\title{
IMPORTANT ANNOUNCEMENT
}

Due to the increased flow of excellent papers from research workers and practitioners throughout the world, Law and Human Behavior will be expanding in 1990 , becoming a bimonthly publication with the number of pages published increased by $57 \%$. Thus, the subscription price for Volume 14, 1990 (6 issues) is $\$ 225.00$ (outside the U.S., $\$ 260.00$ ). The price for individuals certifying that the journal is for their personal use is $\$ 39.50$ (outside the U.S., \$50.00). 